\title{
Pancreatic cancer biomarkers discovery by surface-enhanced laser desorption and ionization time-of-flight mass spectrometry ${ }^{11}$
}

\author{
Filippo Navaglia', Paola Fogar², Daniela Basso, \\ Eliana Greco', Andrea Padoan', Loris \\ Tonidandel $^{3}$, Elisa Fadi ${ }^{1}$, Carlo-Federico \\ Zambon², Dania Bozzato', Stefania Moz', \\ Roberta Seraglia ${ }^{3}$, Sergio Pedrazzoli ${ }^{2}$ and \\ Mario Plebani ${ }^{1, *}$ \\ ${ }^{1}$ Department of Laboratory Medicine, University of \\ Padova, Padova, Italy \\ 2 Department of Medical and Surgical Sciences, \\ University of Padova, Padova, Italy \\ ${ }^{3}$ CNR-ISTM, Padova, Italy
}

\begin{abstract}
Background: Surface-enhanced laser desorption and ionization time-of-flight mass spectrometry (SELDITOF/MS), a laboratory-friendly technique, is used to identify biomarkers for cancer. The aim of the present study was to explore the application of SELDI proteomic patterns in serum for distinguishing between cases of pancreatic cancer, chronic pancreatitis, type 2 diabetes mellitus and healthy controls.

Methods: Sera from 12 healthy controls, 24 patients with type 2 diabetes mellitus, 126 with pancreatic cancer, including 84 with diabetes, and 61 with chronic pancreatitis, 32 of which were diabetics, were analyzed using SELDI-TOF/MS. Spectra (IMAC-30) were clustered and classified using Biomarker Wizard and Biomarker Pattern software.

Results: Two decision tree classification algorithms, one with and one without CA 19-9, were constructed. In the absence of CA 19-9, the splitting protein peaks were: $\mathrm{m} / \mathrm{z}$ 1526, 1211, and 3519; when CA 19-9 was used in the analysis, it replaced the $\mathrm{m} / \mathrm{z} 3519$ splitter. The two algorithms performed equally for classifying patients. A classification tree that considered diabetic patients only was constructed; the main splitters were: 1211, CA $19-9,7903,3359,1802$. With this algorithm, $100 \%$ of patients with type 2 diabetes mellitus, $97 \%$ with chronic pancreatitis and $77 \%$ of patients with pancreatic cancer were correctly classified. SELDI-TOF/MS features improved the diagnostic accuracy of CA 19-9 (AUC $=0.883$ for $C A 19-9 ; A \cup C=0.935$ for CA $19-9$ and SELDI-TOF/MS features combined).
\end{abstract}

\footnotetext{
1) The article is based on a contribution at the 4th Santorini Conference Biologie Prospective, September 21-23, 2008. *Corresponding author: Professor Mario Plebani,

Department of Laboratory Medicine, University-Hospital of Padova, Via Giustiniani 2, 35128 Padova, Italy Phone: + 390498212792, Fax: +39049663240,

E-mail: mario.plebani@unipd.it

Received November 13, 2008; accepted March 24, 2009; previously published online May 11, 2009
}

Conclusions: SELDI-TOF/MS allows identification of new peptides which, in addition to CA 19-9, enable the correct classification of the vast majority of patients with pancreatic cancer, which can be distinguished from patients with chronic pancreatitis or type 2 diabetes mellitus.

Clin Chem Lab Med 2009;47:713-23.

Keywords: chronic pancreatitis; diabetes mellitus; mass spectrometry (MS); pancreatic cancer; surfaceenhanced laser desorption and ionization time-offlight mass spectrometry (SELDI-TOF/MS).

\section{Introduction}

Pancreatic adenocarcinoma has a very unfavorable prognosis with overall 5 years survival not exceeding $5 \%$. In 2008, pancreatic adenocarcinoma accounted for over an estimated 34,000 deaths in the USA alone (1). This high mortality rate is primarily attributed to late diagnosis and to lack of effective chemotherapy. Currently, surgery is the only curative approach available for this tumor, although a limited number of patients, $\sim 20 \%$, have resectable tumor at diagnosis (2). Improvement in survival might be obtained by increasing the number of candidates for curative surgery, and this objective could be accomplished by establishing a diagnosis as early as possible. The identification of patients affected by early pancreatic adenocarcinoma cannot be based exclusively on symptoms which, in the majority of the cases, are unremarkable and similar to those of functional gastrointestinal disorders including dyspepsia, abdominal discomfort, and decreased appetite. Clinical features, such as jaundice or weight loss, which usually occur at more advanced stages, compromise the efficacy of curative surgery.

One major clinical feature of pancreatic cancer is early onset diabetes mellitus or reduced glucose tolerance, diagnosed in more than $80 \%$ of patients (3-5). This is thought to occur as a consequence of release of low molecular weight diabetogenic peptides by cancer cells $(6,7)$. The identification of cancer-associated diabetogenic biomarkers in sera may be extremely useful in identifying patients with newly diagnosed diabetes mellitus as a consequence of underlying pancreatic cancer.

Of the numerous biomarkers studied in patients with pancreatic cancer, the only one currently recommended in clinical practice is serum CA 19-9. However, this marker is of little utility in establishing an 
early diagnosis (8). In fact, circulating concentrations of this marker parallel tumor stage and may be altered in cases of biliary obstruction (9). This makes it difficult to establish whether an increased CA 19-9 concentration in a jaundiced patient is due to the presence of pancreatic malignancy, or due to benign biliary obstruction. Thus, new diagnostic modalities that enable early detection of pancreatic cancer in a safe, non-invasive and cost-effective way are urgently needed.

The discovery of new cancer biomarkers has received renewed interest in recent years as a result of advances in the field of proteomics. The proteome is believed to hold secrets to many patho-physiological alterations, including those associated with cancer (10-12). Serum or plasma are suitable materials for studying the proteome in the attempt to identify new cancer biomarkers. However, proteins present in high abundance such as albumin and immunoglobulins might obscure the identification of potentially new biomarkers that are present in low abundance $(13,14)$. Sera from patients with cancer, including that from patients with pancreatic cancer, usually have a rich protein profile in the low molecular weight range ( $<15,000 \mathrm{Da}$ ) (15-21). The enrichment of low molecular weight peptides in sera from cancer patients may occur as a consequence of direct production by the tumor, or it may be due to expression of a biological cascade of enzymatically-generated and proteolytically-cleaved biomarker fragments. Since major serum proteins have molecular weights much higher than $15,000 \mathrm{Da}$, their interference might be limited when the search for new biomarkers is focused on proteins of low molecular weight. Study of the low molecular weight range serum proteome can be performed using different mass spectrometry (MS) based proteomic techniques that measure the mass to charge $(\mathrm{m} / \mathrm{z})$ ratio of ionizing particles including small molecules, protein/peptides, and fatty acids (12). MS techniques such as matrix assisted laser desorption/ ionization-time-of-flight (MALDI-TOF), electrospray ionization (ESI), QSTAR-TOF and surface-enhanced laser desorption and ionization (SELDI)-TOF may be employed to study the serum proteome, including low molecular weight peptides. SELDI-TOF/MS has an advantage over the other procedures in that it is a high throughput technique which facilitates the identification of multiple biomarkers $(10,12,14)$. In addition, it is considered one of the most useful tools available for the analysis of serum or plasma. Crude samples can be applied directly to an affinity surface, and highly abundant proteins are cleaned from unbound proteins and other interfering substances by repeated washing. As a result of these characteristics, SELDI-TOF/MS is a laboratory friendly, rapid procedure suitable for analysis of large numbers of samples.

The aim of the present study was to identify, using SELDI-TOF/MS, serum specific peptide biomarkers able to differentiate patients with pancreatic adenocarcinoma from those with diabetes mellitus or chronic pancreatitis, and from healthy controls.

\section{Materials and methods}

\section{Patients and samples}

Two hundred and twenty-three subjects participated in the study and their clinical characteristics are detailed in Table 1.

Table 1 Clinical characteristics of the 4 subjects' groups included in the study.

\begin{tabular}{|c|c|c|c|c|}
\hline & $\begin{array}{l}\text { Pancreatic } \\
\text { cancer }\end{array}$ & $\begin{array}{l}\text { Chronic } \\
\text { pancreatitis }\end{array}$ & $\begin{array}{l}\text { Type } 2 \\
\text { diabetes } \\
\text { mellitus }\end{array}$ & $\begin{array}{l}\text { Healthy } \\
\text { controls }\end{array}$ \\
\hline $\begin{array}{l}\text { Total number of cases } \\
\text { Gender (cases) }\end{array}$ & 126 & 61 & 24 & 12 \\
\hline Males & 72 & 47 & 12 & 2 \\
\hline Females & 54 & 14 & 12 & 10 \\
\hline Statistics & \multicolumn{4}{|c|}{$\chi^{2}=18, p<0.001$} \\
\hline \multicolumn{5}{|l|}{ Age (years) } \\
\hline Mean & 67 & 56 & 55 & 41 \\
\hline Median & 69 & 56 & 56 & 39 \\
\hline Range & $43-86$ & $18-81$ & $26-78$ & $29-52$ \\
\hline 25th percentile & 60 & 50 & 44 & 35 \\
\hline 75th percentile & 75 & 66 & 71 & 49 \\
\hline Statistics & \multicolumn{4}{|c|}{ Kruskal-Wallis test: $\chi^{2}=60, p<0.001$} \\
\hline \multicolumn{5}{|l|}{ Comorbidity (cases no.) } \\
\hline Diabetes mellitus & 79 & 30 & 24 & 0 \\
\hline RGT & 12 & 3 & 0 & 0 \\
\hline Normal & 35 & 28 & 0 & 12 \\
\hline Statistics & \multicolumn{4}{|c|}{$\chi^{2}=46, p<0.001$} \\
\hline \multicolumn{5}{|l|}{ CA $19-9$ (kU/L) } \\
\hline Mean & 25,133 & 29 & 13 & 14 \\
\hline Median & 235 & 11 & 11 & 11 \\
\hline Range & $1-1.3 \times 10^{6}$ & $1-307$ & 0-39 & $2-32$ \\
\hline 25th percentile & 90 & 6 & 6 & 8 \\
\hline 75th percentile & 972 & 30 & 19 & 21 \\
\hline Statistics & \multicolumn{4}{|c|}{ Kruskal-Wallis test: $\chi^{2}=86, p<0.001$} \\
\hline
\end{tabular}

RGT, reduced glucose tolerance. 
For all cases, the diagnosis of pancreatic adenocarcinoma was confirmed histologically on surgical specimens. Tumor stages were: IB (9 cases), IIA (11), IIB (37), III (25), and IV (44). Of the 126 patients with pancreatic cancer, 94 had a median follow-up of 11.5 (range 1-131) months. Chronic pancreatitis was diagnosed based on positive findings from at least two of the following: plain abdomen X-ray for pancreatic calcifications; pancreatic ultrasonography; computed axial tomography; endoscopic retrograde pancreatography. Diagnosis of diabetes mellitus was based upon a fasting plasma glucose concentration $>7.0 \mathrm{mmol} / \mathrm{L}$ on two separate days, or a 2-h post-load glucose concentration above $11.1 \mathrm{mmol} / \mathrm{L}$ during an oral glucose tolerance test (OGTT). Patients were considered to have reduced glucose tolerance if their fasting plasma glucose levels were above $5.6 \mathrm{mmol} / \mathrm{L}$, but below $7.0 \mathrm{mmol} / \mathrm{L}$, or 2 -h values during an OGTT were above $7.8 \mathrm{mmol} / \mathrm{L}$, but below $11.1 \mathrm{mmol} / \mathrm{L}$ (22). Using these criteria, overt diabetes mellitus and reduced glucose tolerance were diagnosed in a subset of patients with pancreatic cancer and chronic pancreatitis (Table 1). A fasting blood sample was obtained from all subjects. Each sample was allowed to clot and then centrifuged at $4000 \times g$ for $5 \mathrm{~min}$. The sera was collected and stored at $-20^{\circ} \mathrm{C}$ until examination.

\section{SELDI-TOF/MS analysis}

Ten microliter of each serum sample was applied to IMAC30 ProteinChip array surface (Bio-Rad Laboratories, Hercules, CA, USA), a metal affinity capture. Following a coupling reaction with copper $\left(5 \mu \mathrm{L} \mathrm{CuSO}_{4} 0.1 \mathrm{M}\right.$ surface treatment for $10 \mathrm{~min}$ at room temperature), samples were washed, treated with sodium acetate $(0.1 \mathrm{M} \mathrm{pH} 4.0)$, re-washed and treated with binding buffer (PBS $50 \mathrm{mM}, \mathrm{pH}$ 7.2). Samples were then incubated in a humidity chamber for $1 \mathrm{~h}$ at room temperature. Following three more washes with the binding buffer and two washes with distilled water, the arrays were air-dried and applied to the matrix [1.5 $\mu \mathrm{L} \alpha$-Cyano-4hydroxycinnamic acid (HCCA) in 50\% acetonitrile/0.1\% TFA]. Mass spectra were generated with SELDI-TOF/MS using a ProteinChip reader PBSIlc (Bio-Rad Laboratories). The instrument was set to measure in the range between $\mathrm{m} / \mathrm{z} 1000$ and $\mathrm{m} / \mathrm{z} 15,000$. The laser intensity was 120 and detector sensitivity was 6 . External calibration of the instrument was performed using the All-in-1 peptide molecular mass standard (Bio-Rad Laboratories). All samples were normalized according to the total ion current mass-to-charge ratio $(\mathrm{m} / \mathrm{z})$ between 1000 and 15,000 Da, and qualified mass peaks (signal-to-noise ratio $>5$ ) with $\mathrm{m} / \mathrm{z}$ between 1000 and 15,000 Da automatically detected. A peak threshold of $5 \%$ was selected for all spectra. A second-pass peak selection was performed by choosing a mass window within $0.3 \%$ (signal-to-noise ratio $>2$ ), and estimated peaks were added.

\section{Quantitative analysis of human CA 19-9}

Measurement of CA 19-9 serum concentrations were performed using a chemiluminescence procedure (ADVIA Centaur $^{\circledR}$ CP Immunoassay System, Siemens, Deerfield, IL, USA). Briefly, the ADVIA Centaur CA 19-9 assay, a fully automated two-step sandwich immunoassay using direct chemiluminescent technology, eliminates any high dose hook effect. A single monoclonal antibody (116-NS-19-9) is coupled to paramagnetic particles (Solid Phase) and labeled with acridinium ester in the Lite Reagent. After washing, the sample, first incubated with the Solid Phase, is incubated with the Lite Reagent, which develops a chemiluminescent signal directly correlated with the amount of CA 19-9 in the serum.

\section{Protein identification by MALDI-TOF/MS analysis}

Serum samples, diluted 1:2 (v:v) in saline solution, were centrifuged for $1 \mathrm{~h}$ at $6200 \times g$ using Centricon tubes (Millipore, Billerica, MA, USA) at a 50,000 Da cut-off, and for $20 \mathrm{~min}$ at $6200 \times g$ using Centricon tubes at a 10,000 cut-off. Prior to MALDI-TOF/MS analysis, the filtrates were diluted 1:10 with water containing $0.1 \%$ TFA, then desalted and purified using ZipTip $\mathrm{C}_{18}$ pipette tips (Millipore), following the manufacturer's instructions. The low molecular weight peptides were eluted with a solution of HCCA $\left(2.5 \mathrm{~g} / \mathrm{L} \mathrm{H}_{2} \mathrm{O} /\right.$ Acetonitrile 50 / $50 \mathrm{v}: \mathrm{v}$ with $0.1 \%$ TFA). One microliter of the elution mixture was deposited on the stainless steel sample holder, allowed to dry and then introduced into the mass spectrometer. MALDI-TOF/MS analysis was performed using a 4800 MALDI-TOF-TOF instrument (Applied Biosystems, Foster City, CA, USA), operating in reflectron positive ion mode. lons were formed with a pulsed UV laser beam $(\lambda=337 \mathrm{~nm})$. The following experimental conditions were used: source 1: $20 \mathrm{kV}$; grid 1: $16 \mathrm{kV}$; mirror 2: $20.520 \mathrm{kV}$. External mass calibration was performed using Calibration Mixture 2 of Sequazyme Peptide Mass Standards Kit (Applied Biosystems). MS/MS analysis were performed by accelerating the precursor ions to $8 \mathrm{kV}$, and MS/MS spectra were acquired using $2 \mathrm{kV}$ collision energy with CID gas (air) at a pressure of $1.5 \times 10^{-6}$ torr.

\section{Statistical analysis}

ProteinChip Software, version 3.2.1, and its Biomarker Wizard utility 3.1 (Bio-Rad Laboratories) were used to collect, normalize and export instrument data. Student's t-test for unpaired data, one-way ANOVA, Bonferroni's test for pairwise comparisons and Cox regression analysis were performed using SPSS statistical software (version 9.0; Chicago, IL, USA). Biomarker Patterns ${ }^{\text {TM }}$ Software (Bio-Rad Laboratories) was used to construct decision trees. This software allows construction of trees using a CART methodology that consists of binary recursive partitioning (23). This process iterates a binary split. Every parent node is split into two child nodes with a recursive algorithm, treating each child node as a parent. Ten-fold cross-validation was used. The Biomarker Patterns ${ }^{\mathrm{TM}}$ Software allows a receiver operating characteristics (ROC) curve to be obtained from the classification tree. Each point of the curve represents sensitivity and 1-specificity, obtained by considering one terminal node, two terminal nodes, three terminal nodes, and so on, until one less the total number of terminal nodes is reached (23).

\section{Results}

\section{SELDI-TOF/MS disease associated features}

Following the analysis of all spectra, 219 peak clusters were identified with an $\mathrm{m} / \mathrm{z}$ ratio ranging from a minimum of 1007 to a maximum of 9255 . This range covered the low molecular weight range of the human serum proteome. Univariate analysis revealed that the peak intensities of the four patient groups studied were significantly different for $165 / 219$ features. Some features were correlated with type 2 diabetes mellitus, while others were found in abundance primarily in patients with pancreatic cancer or chronic pancreatitis. Several features were frequently altered in all patients when compared with controls (Table 2 and Supplementary Table 1). Of the 12 features associated with chronic pancreatitis, 10 had an $\mathrm{m} / \mathrm{z}$ within the 
Table 2 Pancreatic cancer associated SELDI-TOF/MS features.

\begin{tabular}{|c|c|c|c|c|c|}
\hline $\mathrm{m} / \mathrm{z}$ & CS & PC & $\mathrm{CP}$ & DM & One-way ANOVA \\
\hline \multicolumn{6}{|c|}{ Features associated with pancreatic cancer } \\
\hline 1542 & $4.8 \pm 0.5$ & $24.5 \pm 1.7^{\mathrm{a}}$ & $17.4 \pm 2.4$ & $18.1 \pm 2.2$ & $F=6.0, p<0.05$ \\
\hline 1637 & $1.9 \pm 0.3$ & $13.3 \pm 0.9^{a}$ & $8.4 \pm 0.9$ & $8.6 \pm 0.8$ & $F=10.3, p<0.001$ \\
\hline 1802 & $1.9 \pm 0.5$ & $19.6 \pm 1.3^{\mathrm{b}}$ & $11.7 \pm 1.7$ & $8.7 \pm 0.9$ & $F=12.3, p<0.001$ \\
\hline 1805 & $0.7 \pm 0.3$ & $18.2 \pm 1.4^{\mathrm{a}, \mathrm{c}}$ & $11.4 \pm 1.2$ & $10.7 \pm 2.5$ & $F=9.6, p<0.001$ \\
\hline 1916 & $3.0 \pm 1.2$ & $12.9 \pm 0.9^{\mathrm{a}}$ & $9.8 \pm 1.1$ & $10.2 \pm 1.5$ & $F=5.6, p<0.01$ \\
\hline 1977 & $6.6 \pm 0.9$ & $12.7 \pm 1.4^{c}$ & $4.4 \pm 0.8$ & $8.7 \pm 1.4$ & $\mathrm{~F}=6.2, \mathrm{p}<0.001$ \\
\hline 2140 & $1.2 \pm 0.3$ & $8.0 \pm 0.8^{a}$ & $6.0 \pm 1.0$ & $6.1 \pm 0.9$ & $F=3.3, p<0.05$ \\
\hline 2582 & $1.1 \pm 0.4$ & $4.4 \pm 0.4^{\mathrm{b}}$ & $2.4 \pm 0.3$ & $1.8 \pm 0.4$ & $F=7.4, p<0.001$ \\
\hline 2878 & $1.1 \pm 0.1$ & $5.3 \pm 0.4^{a, c}$ & $3.4 \pm 0.4$ & $4.0 \pm 0.4$ & $F=6.7, p<0.001$ \\
\hline 3519 & $0.4 \pm 0.1$ & $3.1 \pm 0.3^{a, c}$ & $1.6 \pm 0.3$ & $2.4 \pm 0.3$ & $F=6.6, p<0.01$ \\
\hline 3756 & $1.6 \pm 0.5$ & $8.4 \pm 0.8^{\mathrm{a}, \mathrm{d}}$ & $5.8 \pm 0.8$ & $0.9 \pm 0.1$ & $\mathrm{~F}=8.6, \mathrm{p}<0.01$ \\
\hline 4058 & $2.2 \pm 0.2$ & $10.9 \pm 0.9^{a}$ & $7.2 \pm 0.9$ & $7.8 \pm 1.7$ & $F=5.0, p<0.01$ \\
\hline 4265 & $5.2 \pm 0.9$ & $12.2 \pm 1.0^{\mathrm{a}, \mathrm{d}}$ & $6.5 \pm 0.5$ & $4.8 \pm 0.6$ & $\mathrm{~F}=9.2, \mathrm{p}<0.001$ \\
\hline
\end{tabular}

The results of the univariate analysis (one-way ANOVA) are reported. The following groups were considered: healthy controls (CS), pancreatic cancer (PC), chronic pancreatitis (CP) and type 2 diabetes mellitus (DM). Mean \pm standard error (SE) of peak intensity for each group is reported. Bonferroni's test for pairwise comparisons: ${ }^{a}$ with respect to controls; ${ }^{b}$ with respect to all the others; ${ }^{c}$ with respect to chronic pancreatitis; ${ }^{d}$ with respect to type 2 diabetes mellitus.

range 2000-3000. Nineteen of the 24 features associated with type 2 diabetes had an $\mathrm{m} / \mathrm{z}$ within the range 1000-2000. Although no feature specific for pancreatic cancer was identified, some features were found in greater abundance in patients with tumors, and $4 / 13$ patients had an $\mathrm{m} / \mathrm{z}$ over 3000 . Figure 1 illustrates a typical mass spectra from a patient with pancreatic cancer, a patient with chronic pancreatitis, one with type 2 diabetes mellitus and a healthy control.
The 219 features that were identified were then used to construct the decision classification tree algorithm to identify clusters of features allowing patients with pancreatic cancer to be discriminated from patients with chronic pancreatitis, those with type 2 diabetes mellitus, and healthy controls. The model with the highest classification accuracy was constructed using three features (m/z 1526, 1211 and 3519) to generate four terminal nodes (Figure 2). According to the tree

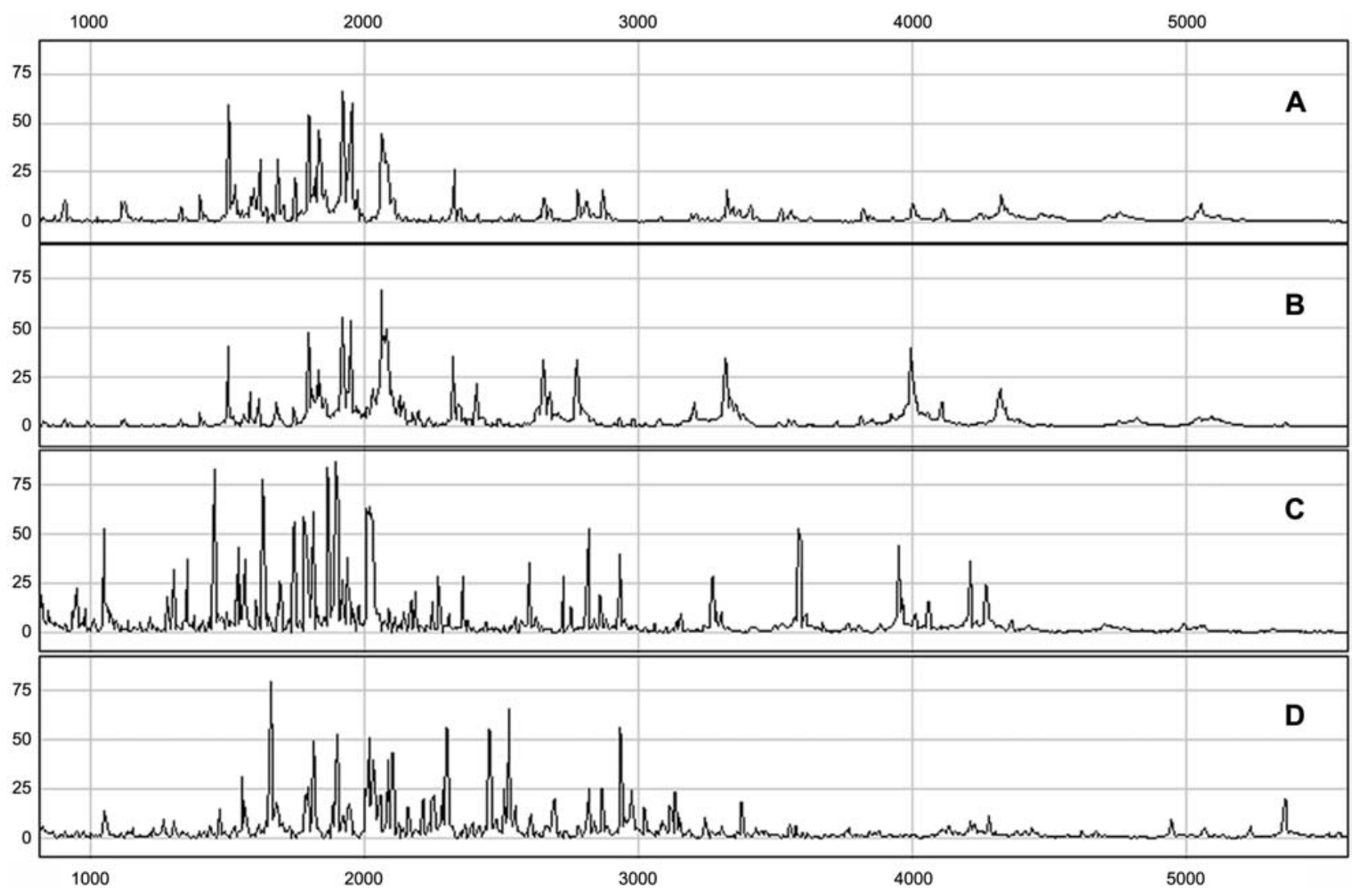

Figure 1 SELDI-TOF/MS spectra obtained from one patient with chronic pancreatitis (A), one with pancreatic cancer (B), one with type 2 diabetes mellitus (C) and one healthy control (panel D).

$\mathrm{m} / \mathrm{z}$ values are reported on the $\mathrm{x}$-axis, and peak intensities on the $\mathrm{y}$-axis. 


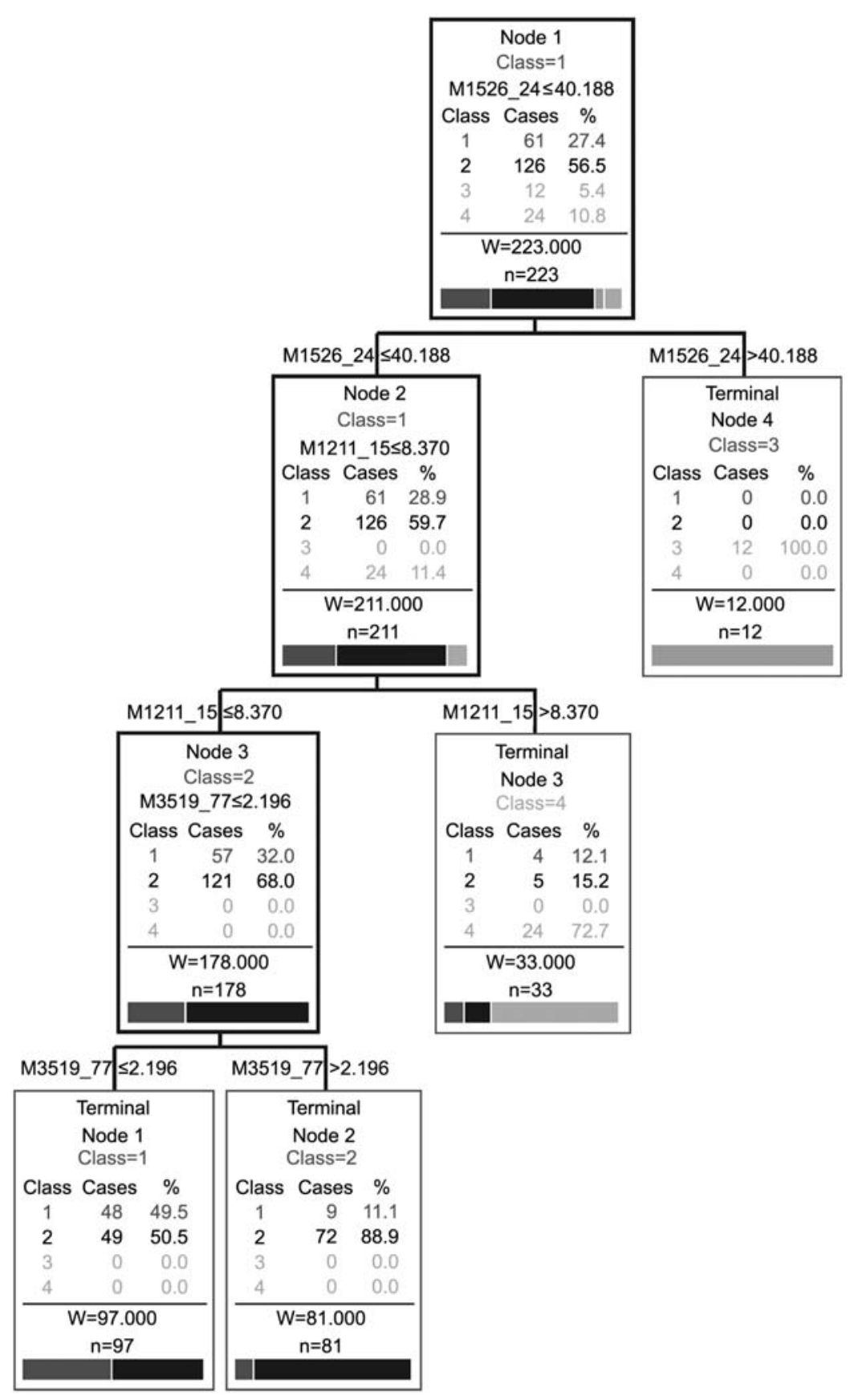

Figure 2 Classification algorithms obtained using SELDI-TOF/MS features.

Class 1, chronic pancreatitis; class 2, pancreatic cancer; class 3, healthy controls; class 4, type 2 diabetes mellitus.

classification model, if an unknown sample had an $\mathrm{m} / \mathrm{z}$ of 1526 with a peak intensity of $>40,188$, it was placed in terminal node 4 and classified as a healthy control (percentage of correct classification $=100$ ); otherwise it was placed in the left branch node. The cases in the left branch node were re-classified according to the peak intensity of the second main splitter, at $\mathrm{m} / \mathrm{z}$ 1211. If the intensity was $>8.37$, samples were placed in terminal node 3 and considered type 2 diabetes mellitus (percentage of correct classification $=72.7$ ). However, if the intensity was $\leq 8.37$, they were placed in the left branch and further subdivided on the basis of the intensity of the third main splitter at $\mathrm{m} / \mathrm{z}$ 3519. If the latter peak intensity was $>2.196$, cases were placed in terminal node 2 and classified as pancreatic cancer (percentage of correct classification $=88.9$ ); otherwise, they were placed in the terminal node 1 and classified as chronic pancreatitis (percentage of correct classification $=49.5$ ). The overall accuracy of this classification tree was $69.9 \%$.

\section{Comparison of SELDI-TOF/MS disease associated features with CA 19-9}

To improve the detection rate, serum CA 19-9 concentrations were measured in all individuals who had adequate residual sample for analysis (12 healthy controls, 24 type 2 diabetes mellitus patients, 42 patients with chronic pancreatitis, and 114 with pancreatic cancer). This biomarker was used together 
with the 219 SELDI-TOF/MS features to construct another decision classification tree. The best model obtained confirmed the two SELDI-TOF/MS main splitters at $\mathrm{m} / \mathrm{z} 1526$ and $\mathrm{m} / \mathrm{z}$ 1211. The third splitter (m/z 3519) was replaced by CA 19-9 with a discriminant value of $111 \mathrm{kU} / \mathrm{L}$. Combining CA 19-9 and SELDI-TOF/MS classifiers showed a higher percentage of patients with pancreatic cancer or chronic pancreatitis being classified correctly $(96.4 \%$ of pancreatic cancer cases in terminal node 2 and $56.8 \%$ of chronic pancreatitis cases in terminal node 1), with an overall accuracy of $76.2 \%$ being achieved. Using the established cut-off of $37 \mathrm{kU} / \mathrm{L}, \mathrm{CA} 19-9$ alone had a sensitivity of $82.8 \%$ in diagnosing pancreatic cancer, a specificity of $100 \%$ with respect to healthy controls, $83.3 \%$ with respect to chronic pancreatitis and $95.5 \%$ with respect to type 2 diabetes mellitus. Its overall accuracy was $85.7 \%$. SELDI-TOF/MS classifiers did not improve the overall diagnostic performance of CA 199 alone in diagnosing patients with pancreatic cancer [area under the ROC curve (AUC) $=0.889$ for CA 19-9; $A U C=0.812$ for SELDI-TOF/MS features; $A U C=0.860$ for CA 19-9 and SELDI-TOF/MS features combined]. Therefore, all subsequent analyses included CA 19-9.

\section{SELDI-TOF/MS disease associated features and CA 19-9 in patients with diabetes mellitus}

One of the objectives of the present study was to identify new biomarkers that would allow more accurate distinctions to be made between patients with diabetes mellitus with and without pancreatic cancer. We selected only cases with diabetes mellitus and available CA 19-9 levels. This gave us 24 patients with type 2 diabetes mellitus, 29 with chronic pancreatitis and 73 with pancreatic cancer. The diagnostic efficacy of CA 19-9 alone and SELDI-TOF/MS classifiers alone was similar, but when both parameters were combined, significant improvement was achieved in discriminating between pancreatic cancer and chronic pancreatitis or patients with type 2 diabetes mellitus (Figure 3). The algorithm obtained by combining SELDI-TOF/MS features and CA 19-9 (Figure 4) allowed correct classification of almost all cases with type 2 diabetes mellitus (100\% correct classification). However, it did not allow us to obtain definite discrimination of pancreatic cancer $(76.7 \%$ correct classification) and chronic pancreatitis cases $(96.6 \%$ correct classification).

\section{Metastases and survival of pancreatic cancer patients: SELDI-TOF/MS features and CA 19-9}

The presence or absence of metastases was considered in pancreatic cancer cases in order to construct a new algorithm (Figure 5). CA 19-9 was selected in this case as the main splitter, together with the feature at $\mathrm{m} / \mathrm{z} 3199$. Forty of the 74 cases without metastases were allocated to terminal node 1 (absence of metastases) on the basis of CA 19-9 concentrations $\leq 220.6 \mathrm{kU} / \mathrm{L}$. For the 66 cases with CA $19-9$ concen-

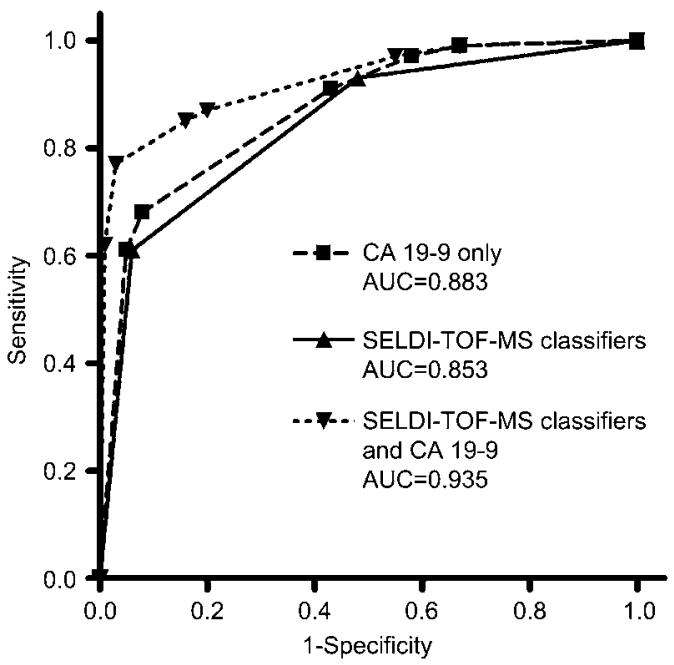

Figure 3 Receiver operating characteristic (ROC) curves and areas under the curves (AUC) showing the discriminating capacity of SELDI-TOF/MS classifiers and CA 19-9 individually and combined, considering only patients with diabetes mellitus (24 type 2 diabetes mellitus, 29 chronic pancreatitis, 73 pancreatic cancer).

trations $>220.6 \mathrm{kU} / \mathrm{L}$, the second splitter $(\mathrm{m} / \mathrm{z}$ 3199) allowed the identification of metastases, with a sensitivity of $100 \%$ and a specificity of $35 \%$.

In order to investigate whether SELDI-TOF/MS features were predictive of survival in patients with pancreatic cancer, an analysis was made of the 94 patients who had follow-up following diagnosis. Patients were subdivided into two groups on the basis of survival: short-term ( $\leq 18$ months) and long-term survivors (>18 months). Six SELDI-TOF/MS features significantly correlated with survival (Table 3 ), which did not correlate with CA 19-9 $(t=1.5, p: n s)$. We selected the six significant features reported in Table 3 as covariates in order to perform Cox regression analysis, patients being stratified on the basis of tumor stage. Only three features were confirmed to be significantly correlated with survival, those at $\mathrm{m} / \mathrm{z}$ 1805, 2054 and 2878.

\section{MALDI-TOF/MS analysis}

Sera with a high abundance of features at $\mathrm{m} / \mathrm{z} 1526$, 1211 and 3519, the main splitters of the diagnostic tree algorithm, were selected and analyzed using MALDITOF/MS to confirm their presence in the samples. Since SELDI-TOF/MS has a lower mass resolution compared with MALDI-TOF/MS, a perfect mass correspondence between the $\mathrm{m} / \mathrm{z}$ values of ions detected by the two analytical instruments cannot be expected. In MALDI-TOF/MS spectra, the ion at m/z 1526 was not detected, while the ionic species at $\mathrm{m} / \mathrm{z} 1211$ and $\mathrm{m} / \mathrm{z} 3519$ (corresponding to MALDI-TOF/MS value: $\mathrm{m} / \mathrm{z}$ 3522.2080) were detected with a very low abundance (Figure 6, panel A). The only MS/MS spectrum useful for obtaining peptide sequence information was achieved for the $\mathrm{m} / \mathrm{z} 3522.2080$ ionic specie (Figure 6, panel B). A match search using Protein Prospector 


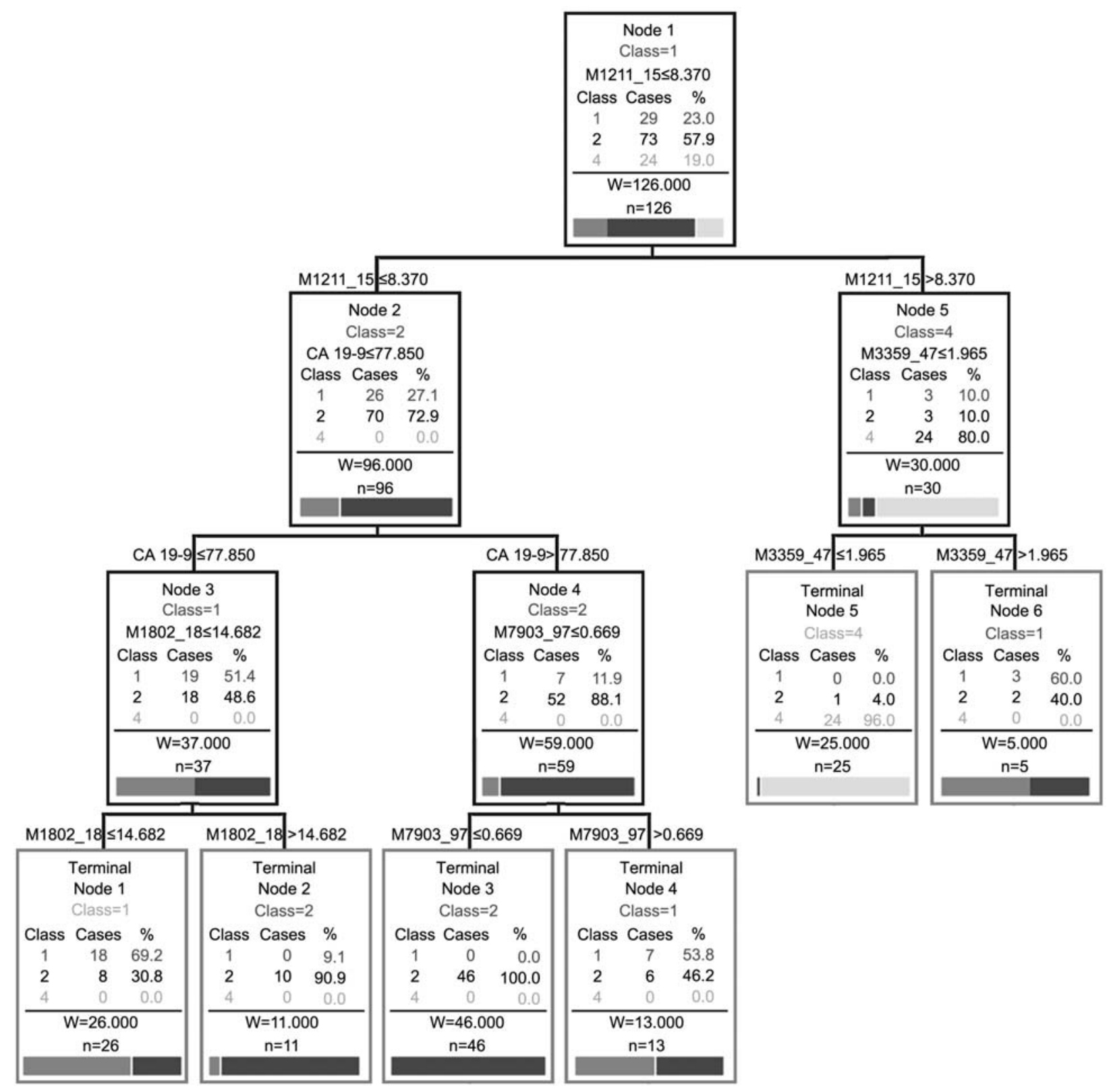

Figure 4 Classification algorithm obtained using SELDI-TOF/MS features and CA 19-9 combined, considering only patients with diabetes ( 24 type 2 diabetes mellitus, 29 chronic pancreatitis, 73 pancreatic cancer).

Class 1, chronic pancreatitis; class 2, pancreatic cancer; class 4, type 2 diabetes mellitus.

version 4.27.2 in the database Swiss Prot 2008.06.10 was performed. A series of possible putative protein fragments were identified; among them, the most reliable was the deamidated fragment $458-493$ of Multiple Epidermal Growth Factor-like domain 6 precursor [N(Deamidated)CSFSCSCON(Deamidated)GGTCDSVTGACRCPPGVSGTN(Deamidated)CEDGC; NCBI accession number: 075095] (m/z 3522.2082; number of matching fragments $=27 / 36$; probability score =21.4). Few peaks due to the main sequence ions (i.e., $\mathrm{y}$ - and b-series) were present in the MS/MS spectrum of this peptide. The majority of the peaks originated from internal cleavages. This pattern can be ascribed to the presence of three deamidated $\mathrm{N}$ residues. Details on fragments are reported in Supplementary Table 2.

\section{Discussion}

In the present study, the SELDI-TOF/MS proteomic approach was used to identify new low-molecular weight serum biomarkers for the diagnosis of pancreatic cancer. Previous studies focusing on this issue were conducted considering only patients with pancreatic cancer and controls $(16,24)$, or had controls that included few patients with chronic or acute pancreatitis $(18,21,25)$. As yet, no studies are available that have compared the serum proteomic profile of patients with pancreatic cancer with those having type 2 diabetes mellitus. However, it is important to consider patients with both chronic pancreatitis and type 2 diabetes mellitus because: 1) in some cases, the clinical picture of chronic pancreatitis mimics that 


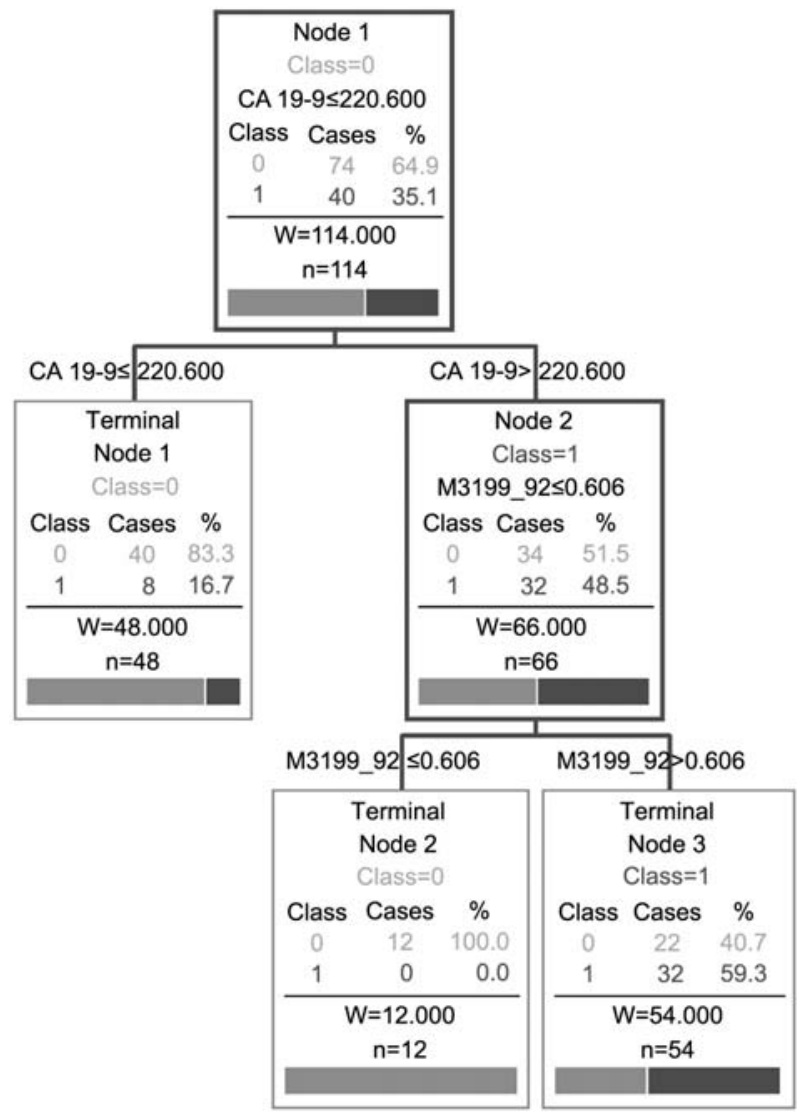

Figure 5 Classification algorithm obtained using SELDITOF/MS features and CA 19-9 combined to discriminate between pancreatic cancer patients with (class 1 ) and those without (class 0 ) distant metastases.

$114 / 126$ pancreatic cancer patients were available for analysis, since in 12 cases CA 19-9 values were unavailable due to lack of material.

of pancreatic cancer, and vice versa; 2) chronic pancreatitis is considered a risk factor for pancreatic cancer, the onset of which should be identified as early as possible $(26) ; 3$ ) in many cases, diabetes mellitus, frequently associated with pancreatic cancer, is the presenting symptom of the latter (3-5). Since SELDITOF/MS provides the opportunity to analyze all the low molecular weight serum proteome (27), we obtained accurate sera profiles within the range of $\mathrm{m} / \mathrm{z} 1000-10,000$. Of the numerous features identified using this range, many were present only occasion- ally. Although their diagnostic and/or pathophysiological significance cannot be ruled out "a priori", we decided to analyze only those features detectable in more than $5 \%$ of spectra. With this limit, a total of 219 features were entered in the analysis of data. For each feature, the abundance, which grossly indicates the amount of a given $\mathrm{m} / \mathrm{z}$ feature, was considered. With univariate analysis, some diabetes-, chronic pancreatitis- and pancreatic cancer-associated features emerged. In addition, many features that are commonly altered in diseased patients were seen. Many features associated with pancreatic cancer were not specifically correlated with this tumor type, but were also present in chronic pancreatitis. This finding is in agreement with results obtained by Kojima et al. (21). These investigators used MALDI-TOF/MS to analyze sera and urine from a series of patients comparable to ours, and found no tumor-specific feature. One of the features identified by these authors as increased in the urine samples of patients with both pancreatic cancer and chronic pancreatitis compared to healthy controls (m/z 2193), was detected by us in the sera of the same groups of patients. However, we observed the opposite findings: the mean abundance was lower in patients than in healthy controls. Other differentially expressed features detected in the present series, particularly those at $\mathrm{m} / \mathrm{z}$ 4277, 3756 and 4058, were identical or very close to features identified by other authors (16). These other investigators not only selected similar patient series and used the SELDI technique, but also employed the same protein chip as that used by us (IMAC30).

The data described above support the concept that the reproducibility of MS in different laboratories calls for similar series of patients to be analyzed using standardized analytical procedures. One feature believed to be associated with pancreatic cancer, $\mathrm{m} / \mathrm{z}$ 4058 , was found by us to be very similar to a feature described by Scarlett et al. (28); concentrations being higher in cyst fluid from three patients with pancreatic cancer compared with cyst fluid obtained from seven patients who were carcinoma-free. This finding, potentially of clinical relevance, supports the $\mathrm{m} / \mathrm{z} 4058$ feature as being a product of pancreatic cancer that can be detected in serum. However, for the vast majority of cancer associated features identified in previous studies analyzing tissue samples or pancreatic juice from humans or murine models (29-36), we

Table 3 SELDI-TOF/MS features and pancreatic cancer patients' survival.

\begin{tabular}{|c|c|c|c|c|c|c|c|c|}
\hline \multirow[t]{2}{*}{$\begin{array}{l}\text { Features, } \\
\mathrm{m} / \mathrm{z}\end{array}$} & \multirow{2}{*}{$\begin{array}{l}\text { Long survivors } \\
>18 \text { months } \\
\text { Mean } \pm \text { SEM }\end{array}$} & \multirow{2}{*}{$\begin{array}{l}\text { Short survivors } \\
\leq 18 \text { months } \\
\text { Mean } \pm \text { SEM }\end{array}$} & \multicolumn{2}{|c|}{$\begin{array}{l}\text { Univariate } \\
\text { Student's t-test }\end{array}$} & \multicolumn{4}{|c|}{$\begin{array}{l}\text { Multivariate } \\
\text { Cox regression analysis }\end{array}$} \\
\hline & & & $t$ & $\mathrm{p}$-Value & $\operatorname{Exp}(B)$ & Lower & Upper & $\mathrm{p}$-Value \\
\hline 1805 & $27.0 \pm 3.4$ & $14.1 \pm 1.4$ & 4.1 & $<0.001$ & 0.98 & 0.96 & 1.00 & $<0.05$ \\
\hline 1887 & $6.6 \pm 0.8$ & $10.0 \pm 0.9$ & 2.7 & $<0.01$ & 1.00 & 0.96 & 1.05 & ns \\
\hline 2054 & $4.0 \pm 0.4$ & $6.4 \pm 0.6$ & 3.2 & $<0.01$ & 1.09 & 1.03 & 1.16 & $<0.01$ \\
\hline 2815 & $8.4 \pm 1.6$ & $14.7 \pm 2.0$ & 2.5 & $<0.05$ & 1.00 & 0.97 & 1.02 & ns \\
\hline 2878 & $4.1 \pm 0.7$ & $6.1 \pm 0.6$ & 2.2 & $<0.05$ & 1.10 & 1.03 & 1.18 & $<0.01$ \\
\hline 4100 & $4.3 \pm 0.9$ & $2.5 \pm 0.2$ & 2.9 & $<0.01$ & 1.02 & 0.91 & 1.13 & ns \\
\hline
\end{tabular}

The results of univariate (Student's t-test for unpaired data) and multivariate (Cox regression analysis) statistical analysis are shown. The multivariate test included term for tumor stage. ns, not significant; SEM, standard error of the mean. 


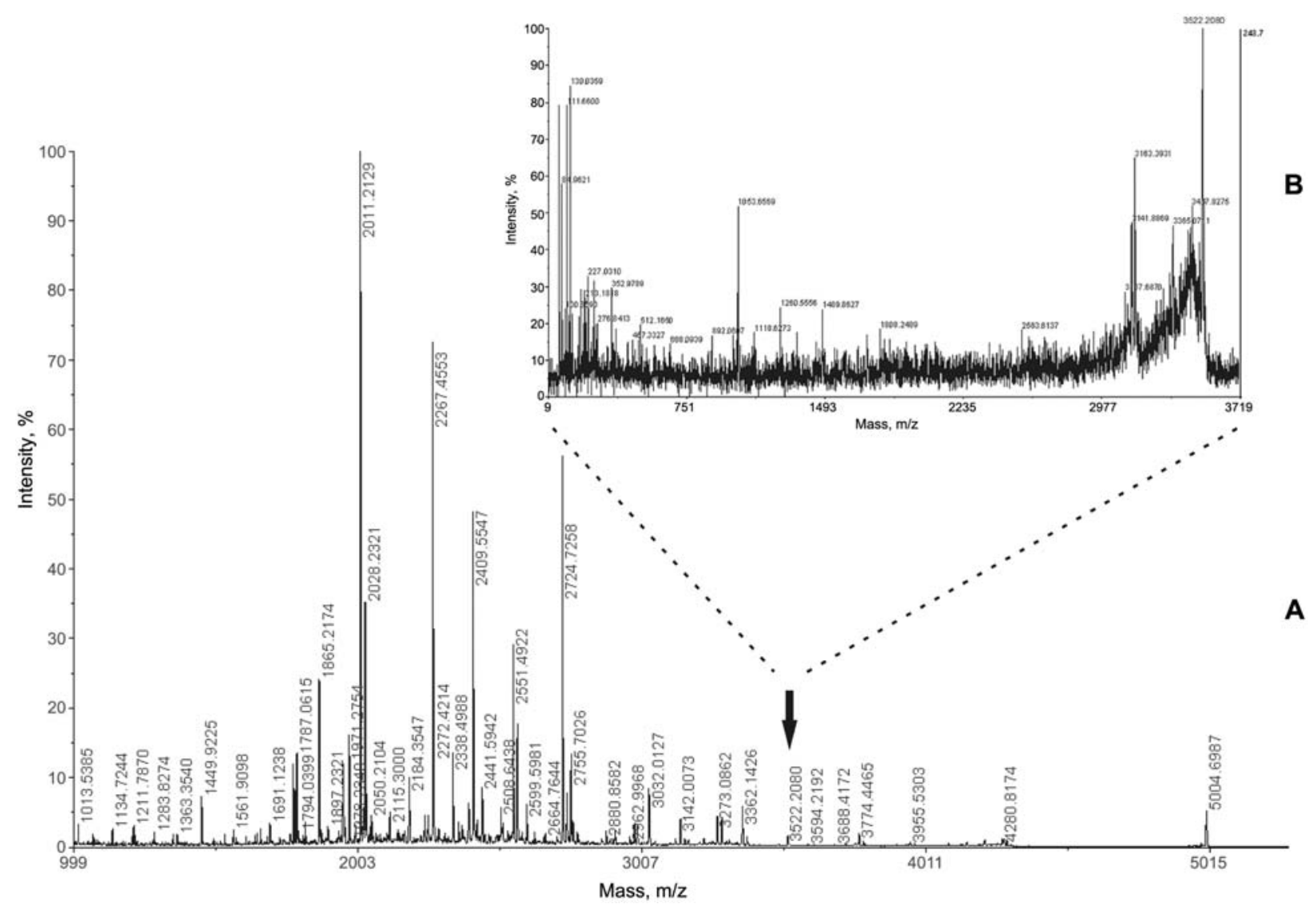

Figure 6 MALDI-TOF/MS serum profile (A) and fragments obtained from the feature at $\mathrm{m} / \mathrm{z} 3522.2080$ (B).

found no match with the features in sera. This apparent discrepancy may be due to differences in the proteomic techniques used in the different studies, and in the clinical selection of patients.

By employing the Biomarker Pattern software, we first generated an algorithm based only on the SELDITOF/MS features. Of the three main splitters selected (m/z 1526, 1211 and 3519), none matched with the previously reported discriminant features obtained using similar protocols $(16,18)$. One of the reasons for this discrepancy may be the presence of patients with type 2 diabetes mellitus in our series. The presence of diabetics has also been suggested by Honda et al. (24) to have a potential role in influencing the classifiers when patients with pancreatic cancer are studied. The 1526 and 1211 features, the main classifiers for type 2 diabetes mellitus, might be metabolic products, proteolytic fragments or peptide hormones $(10,37)$. The overall accuracy of the classification algorithm constructed using SELDI-TOF/MS features only was about $70 \%$. A comparison was then made with the well-established marker CA 19-9, which is widely used in clinical practice for diagnosis, evaluation of therapy and for detecting recurrence $(8,9)$. When used alone, this marker had an overall diagnostic accuracy of $83 \%$, which was not improved when the marker was combined with SELDI-TOF/MS features (76\%). Therefore, SELDI-TOF/MS findings did not reinforce the results obtained with serum CA 19-9 determination alone.
The initial algorithm included patients and healthy controls, who are not usually included in studies on the differential diagnosis of pancreatic cancer. In patients with pancreatic cancer, concomitant pathological conditions such as diabetes mellitus may lead to diagnostic uncertainty. We, therefore, re-evaluated SELDI-TOF/MS features together with CA 19-9, considering only diabetes mellitus patients with pancreatic cancer, chronic pancreatitis or type 2 diabetes mellitus. When considering this cohort of patients, SELDI-TOF/MS features had a diagnostic advantage over CA 19-9 alone, allowing the correct classification of a large percentage of cases (100\% type 2 diabetes mellitus, $76.7 \%$ pancreatic cancer, $96.6 \%$ chronic pancreatitis). However, the distinction made between pancreatic cancer and chronic pancreatitis was weaker than that made between pancreatic cancer and type 2 diabetes mellitus.

Further investigation was done to establish whether SELDI-TOF/MS features might offer insight on some aspects correlated with pancreatic cancer, namely metastases and survival. Only one feature ( $\mathrm{m} / \mathrm{z}$ 3199) was selected, together with CA 19-9, for differentiating between pancreatic cancer patients with and without metastases. No correlation was found with CA 19-9 and, among the 219 SELDI-TOF/MS features, only six were correlated with survival using univariate analysis. Multivariate analysis confirmed that three features, those at $\mathrm{m} / \mathrm{z} 1805,2054$ and 2878, were predictive for pancreatic cancer survival, independent of 
tumor stage. The former and the latter features were associated with pancreatic cancer (Table 1) and appear promising tools for future clinical applications.

Finally, using MALDI-TOF/MS, it was possible to identify the pancreatic cancer-associated ion at $\mathrm{m} / \mathrm{z}$ 3522.2080 as the deamidated fragment $458-493$ of Multiple Epidermal Growth Factor like domains 6 precursor. The Epidermal Growth Factor Family molecules are known to have pro-proliferative action (38), and increased Heparin Binding-EGF like mRNA concentrations have already been described in pancreatic cancer tissue (39). This further supports the idea that this biomarker has potential diagnostic implications.

In conclusion, SELDI-TOF/MS allowed the identification of new peptides in sera. These findings, in addition to CA 19-9, facilitates the differential diagnosis between pancreatic cancer and type 2 diabetes mellitus. Among the large number of classification peptides identified following SELDI-TOF/MS, that at $\mathrm{m} / \mathrm{z}$ 3519, submitted to MALDI-TOF/MS analysis, appears to be a member of the epidermal growth factor like family.

\section{Acknowledgements}

The authors are grateful to Mr. Flaviano Favaro for his technical assistance in measuring serum CA 19-9.

\section{References}

1. Jemal A, Siegel R, Ward E, Hao Y, Xu J, Murray T, et al. Cancer statistics, 2008. CA Cancer J Clin 2008;58:71-96.

2. Pedrazzoli $S$, Pasquali $C$, Sperti $C$. Role of surgery in the treatment of bilio-pancreatic cancer: the European experience. Semin Oncol 2002;29:23-30.

3. Fogar $P$, Pasquali $C$, Basso D, Sperti $C$, Panozzo MP, Tessari $G$, et al. Diabetes mellitus in pancreatic cancer follow-up. Anticancer Res 1994;14:2827-30.

4. Chari ST, Leibson CL, Rabe KG, Timmons LJ, Ransom J, de Andrade $\mathrm{M}$, et al. Pancreatic cancer-associated diabetes mellitus: prevalence and temporal association with diagnosis of cancer. Gastroenterology 2008;134:95-101.

5. Pannala R, Leirness JB, Bamlet WR, Basu A, Petersen GM, Chari ST. Prevalence and clinical profile of pancreatic cancer-associated diabetes mellitus. Gastroenterology 2008;134:981-7.

6. Basso D, Valerio A, Seraglia R, Mazza S, Piva MG, Greco $\mathrm{E}$, et al. Putative pancreatic cancer-associated diabetogenic factor: 2030 MW peptide. Pancreas 2002;24:8-14.

7. Basso D, Greco E, Fogar P, Pucci P, Flagiello A, Baldo G, et al. Pancreatic cancer-derived S-100A8 N-terminal peptide: a diabetes cause? Clin Chim Acta 2006;372:120-8.

8. Locker GY, Hamilton S, Harris J, Jessup JM, Kemeny N, Macdonald JS, et al. ASCO 2006 update of recommendations for the use of tumor markers in gastrointestinal cancer. J Clin Oncol 2006;24:5313-27.

9. Goonetilleke KS, Siriwardena AK. Systematic review of carbohydrate antigen (CA 19-9) as a biochemical marker in the diagnosis of pancreatic cancer. Eur J Surg Oncol 2007;33:266-70.

10. Petricoin EF, Liotta LA. SELDI-TOF-based serum proteomic pattern diagnostics for early detection of cancer. Curr Opin Biotechnol 2004;15:24-30.

11. Cowan ML, Vera J. Proteomics: advances in biomarker discovery. Expert Rev Proteomics 2008;5:21-3.
12. Feng $X$, Liu $X$, Luo $Q$, Liu BF. Mass spectrometry in systems biology: an overview. Mass Spectrom Rev 2008; 27:635-60.

13. Anderson NL, Anderson NG. The human plasma proteome: history, character, and diagnostic prospects. Mol Cell Proteomics 2002;1:845-67.

14. Seibert V, Ebert MP, Buschmann T. Advances in clinical cancer proteomics: SELDI-ToF-mass spectrometry and biomarker discovery. Brief Funct Genomic Proteomic 2005;4:16-26.

15. Valerio A, Basso D, Mazza S, Baldo G, Tiengo A, Pedrazzoli S, et al. Serum protein profiles of patients with pancreatic cancer and chronic pancreatitis: searching for a diagnostic protein pattern. Rapid Commun Mass Spectrom 2001;15:2420-5.

16. Koopmann J, Zhang Z, White N, Rosenzweig J, Fedarko $N$, Jagannath $S$, et al. Serum diagnosis of pancreatic adenocarcinoma using surface-enhanced laser desorption and ionization mass spectrometry. Clin Cancer Res 2004; 10:860-8.

17. Brouwers FM, Petricoin EF 3rd, Ksinantova L, Breza J Rajapakse V, Ross S, et al. Low molecular weight proteomic information distinguishes metastatic from benign pheochromocytoma. Endocr Relat Cancer 2005; 12:263-72.

18. Yu Y, Chen S, Wang LS, Chen WL, Guo WJ, Yan H, et al. Prediction of pancreatic cancer by serum biomarkers using surface-enhanced laser desorption/ionizationbased decision tree classification. Oncology 2005;68: 79-86.

19. Liu XP, Shen J, Li ZF, Yan L, Gu J. A serum proteomic pattern for the detection of colorectal adenocarcinoma using surface enhanced laser desorption and ionization mass spectrometry. Cancer Invest 2006;24:747-53.

20. Hanas JS, Hocker JR, Cheung JY, Larabee JL, Lerner MR, Lightfoot SA, et al. Biomarker identification in human pancreatic cancer sera. Pancreas 2008;36:61-9.

21. Kojima K, Asmellash S, Klug CA, Grizzle WE, Mobley JA, Christein JD. Applying proteomic-based biomarker tools for the accurate diagnosis of pancreatic cancer. J Gastrointest Surg 2008;12:1683-90.

22. American Diabetes Association. Diagnosis and classification of diabetes mellitus. Diabetes Care 2008;31(Suppl 1):55-60.

23. Raubertas RF, Rodewald LE, Humiston SG, Szilagyi PG. ROC curves for classification trees. Med Decis Making 1994;14:169-74.

24. Honda K, Hayashida Y, Umaki T, Okusaka T, Kosuge T, Kikuchi S, et al. Possible detection of pancreatic cancer by plasma protein profiling. Cancer Res 2005;65: 10613-22.

25. Koomen JM, Shih LN, Coombes KR, Li D, Xiao LC, Fidler $\mathrm{IJ}$, et al. Plasma protein profiling for diagnosis of pancreatic cancer reveals the presence of host response proteins. Clin Cancer Res 2005;11:1110-8.

26. Hart AR, Kennedy $H$, Harvey I. Pancreatic cancer: a review of the evidence on causation. Clin Gastroenterol Hepatol 2008;6:275-82.

27. Barbarini N, Magni P, Bellazzi R. A new approach for the analysis of mass spectrometry data for biomarker discovery. AMIA Annu Symp Proc 2006:26-30.

28. Scarlett CJ, Samra JS, Xue A, Baxter RC, Smith RC. Classification of pancreatic cystic lesions using SELDI-TOF mass spectrometry. ANZ J Surg 2007;77:648-53.

29. Rosty C, Christa L, Kuzdzal S, Baldwin WM, Zahurak ML, Carnot $F$, et al. Identification of hepatocarcinoma-intestine-pancreas/pancreatitis-associated protein I as a biomarker for pancreatic ductal adenocarcinoma by protein biochip technology. Cancer Res 2002;62:1868-75.

30. Hingorani SR, Petricoin EF, Maitra A, Rajapakse V, King $C$, Jacobetz MA, et al. Preinvasive and invasive ductal 
pancreatic cancer and its early detection in the mouse. Cancer Cell 2003;4:437-50.

31. Chen R, Yi EC, Donohoe S, Pan S, Eng J, Cooke K, et al. Pancreatic cancer proteome: the proteins that underlie invasion, metastasis, and immunologic escape. Gastroenterology 2005;129:1187-97.

32. Scarlett CJ, Smith RC, Saxby A, Nielsen A, Samra JS, Wilson SR, et al. Proteomic classification of pancreatic adenocarcinoma tissue using protein chip technology. Gastroenterology 2006;130:1670-8.

33. Chen R, Pan S, Cooke K, Moyes KW, Bronner MP, Goodlett $\mathrm{DR}$, et al. Comparison of pancreas juice proteins from cancer versus pancreatitis using quantitative proteomic analysis. Pancreas 2007;34:70-9.

34. Sun ZL, Zhu Y, Wang FQ, Chen R, Peng T, Fan ZN, et al. Serum proteomic-based analysis of pancreatic carcinoma for the identification of potential cancer biomarkers. Biochim Biophys Acta 2007;1774:764-71.
35. Chung JC, Oh MJ, Choi SH, Bae CD. Proteomic analysis to identify biomarker proteins in pancreatic ductal adenocarcinoma. ANZ J Surg 2008;78:245-51.

36. Qi T, Han J, Cui Y, Zong M, Liu X, Zhu B. Comparative proteomic analysis for the detection of biomarkers in pancreatic ductal adenocarcinomas. J Clin Pathol 2008; 61:49-58.

37. Sun S, Lee NP, Poon RT, Fan ST, He QY, Lau GK, et al. Oncoproteomics of hepatocellular carcinoma: from cancer markers' discovery to functional pathways. Liver Int 2007;27:1021-38.

38. Davis-Fleischer KM, Besner GE. Structure and function of heparin-binding EGF-like growth factor (HB-EGF). Front Biosci 1998;3:d288-99.

39. Kobrin MS, Funatomi H, Friess $H$, Buchler MW, Stathis $\mathrm{P}$, Korc M. Induction and expression of heparin-binding EGF-like growth factor in human pancreatic cancer. Biochem Biophys Res Commun 1994;202:1705-9. 